\title{
Statistics Dependence of the Entanglement Entropy
}

\author{
M. Cramer, ${ }^{1,2,3}$ J. Eisert, ${ }^{2,3}$ and M. B. Plenio ${ }^{2,3}$ \\ ${ }^{1}$ Institut für Physik, Universität Potsdam, Am Neuen Palais 10, D-14469 Potsdam, Germany \\ ${ }^{2}$ QOLS, Blackett Laboratory, Imperial College London, Prince Consort Road, London SW7 2BW, United Kingdom \\ ${ }^{3}$ Institute for Mathematical Sciences, Imperial College London, Exhibition Road, London, SW7 2PG, United Kingdom
}

(Received 22 December 2006; revised manuscript received 9 March 2007; published 31 May 2007)

\begin{abstract}
The entanglement entropy of a distinguished region of a quantum many-body system reflects the entanglement in its pure ground state. Here we establish scaling laws for this entanglement in critical quasifree fermionic and bosonic lattice systems, without resorting to numerical means. We consider the setting of $D$-dimensional half-spaces which allows us to exploit a connection to the one-dimensional case. Intriguingly, we find a difference in the scaling properties depending on whether the system is bosonicwhere an area law is proven to hold-or fermionic where we determine a logarithmic correction to the area law, which depends on the topology of the Fermi surface. We find Lifshitz quantum phase transitions accompanied with a nonanalyticity in the prefactor of the leading order term.
\end{abstract}

PACS numbers: 05.50.+q, 03.67.Mn, 05.30. $-\mathrm{d}, 05.70 .-\mathrm{a}$

The occurrence of critical points at zero temperature holds the key to the understanding of several phenomena in quantum many-body systems in the condensed matter context [1]. Quantum criticality is accompanied by a divergence of the typical length scale, the correlation length. These long-range correlations come along with genuine entanglement in the ground state, grasped by the entanglement entropy $E_{S}=S\left(\operatorname{tr}_{\backslash \mathcal{A}}[\rho]\right)$. This is the entropy of the reduced density matrix that is obtained when tracing out the degrees of freedom outside a distinguished region $\mathcal{A}$, quantifying the degree of entanglement between this region and the rest [2-19].

This notion of the entanglement or geometric entropyand more to the point its scaling behavior abstracting from details of the model-has enjoyed a strong revival of interest recently, partially driven by intuition from quantum information theory: previously conjectured scaling laws in higher dimensions [2], relating the entanglement entropy to the boundary area-not the volume-of the region, have been rigorously established using quantum information ideas [4-6]. This was followed by observations of violations of such area laws [7]. The entanglement entropy has in its non-leading-order behavior interestingly been linked to the topology of the system [8], using ideas of topological quantum field theory, and been studied under time evolution [9]. Partly, this renewed interest is triggered by the implications on the simulatability of quantum systems using density-matrix renormalization approaches: the entanglement entropy quantifies in a sense the relevant number of degrees of freedom to be considered [3,10].

If entanglement is to reflect critical or noncritical properties of quantum many-body systems, an area relationship might of course be expected to hold or not, depending on whether the two-point correlation functions diverge. One might be tempted to think that entanglement could yet be seen as an indicator of criticality in the same sense. Intriguingly, it turns out that the situation is more complex.
As we will also see, even for critical systems, an area relationship can hold, despite a divergent correlation length (as can also be observed in projected entangled pair states, satisfying an area law by construction [11]). In this work, we demonstrate with a fully analytical argument that it can depend on the statistics of the system-bosonic or fermionic-whether an area relationship holds or is in fact violated. In this way, we resolve the key open question, What happens in the critical bosonic case? In the process we confirm some conjectures based on numerical findings for small system size $[2,12,13]$ and refute others, such as the conjecture of a breakdown of an area law for critical bosons in $D>1$ in Ref. [14].

Here, we establish first analytical scaling laws for critical bosonic systems. We achieve these results for the geometrical setting of a half-space in $D$ dimensions, completing the program initiated in Ref. [5]. These findings are compared with fermionic half-spaces, complementing recent results on cubic regions in Refs. [7], and in a fashion consistent with numerical work in Refs. $[12,13]$. We treat bosonic and fermionic systems on the same footing - in terms of Majorana operators for fermions and canonical coordinates for bosons. We hence provide a unified and complete framework for entanglement scaling in critical quasifree systems with that geometry.

Setting. - We consider cubic lattices of spatial dimension $D, \mathcal{L}=[1, \ldots, N]^{\times D},|\mathcal{L}|=N^{D}$, equipped with $p e$ riodic boundary conditions and study ground states $\varrho$ of Hamiltonians that are quadratic forms of either bosonic or fermionic operators. The geometric setting is that of a halfspace, distinguishing the first spatial direction, considering a subsystem $\mathcal{A}=[1, \ldots, M] \times \mathcal{L}^{\prime}, \mathcal{L}^{\prime}=[1, \ldots, N]^{\times D-1}$, and its entanglement with the rest $\mathcal{B}=\mathcal{L} \backslash \mathcal{A}=$ $[M+1, \ldots, N] \times \mathcal{L}^{\prime}$. We can hence make use of an idea of exploiting transverse momenta. This geometrical setting has numerically been assessed with respect to local spectra and simulatability issues in the seminal work Ref. [3]. 
When we say that (i) this entanglement as quantified by the von Neumann entropy $E_{S}(M, N)=S\left(\varrho_{\mathcal{A}}\right)$ of the reduced state $\varrho_{\mathcal{A}}=\operatorname{tr}_{\mathcal{B}}[\varrho]$ satisfies an area law, we mean that for $M=N / 2$ the entanglement entropy satisfies

$$
\mathcal{E}:=\lim _{N \rightarrow \infty} E_{S}(M, N) / N^{D-1} \leq \mathrm{const} ;
$$

i.e., it scales at most like the boundary area of $\mathcal{A}$. For systems violating the area law we will (ii) study the exact form of the encountered logarithmic divergence in $M$,

$$
\mathcal{E}=\text { const } \times \log _{2} M+o\left(\log _{2} M\right) .
$$

We will subsequently discuss physical systems that are described by Hamiltonians of the type $\hat{H}=$ $\frac{1}{2} \sum_{i, j}\left[\hat{d}_{i}^{\dagger} A_{i, j} \hat{d}_{j}+\hat{d}_{i} B_{i, j} \hat{d}_{j}^{\dagger}+\hat{d}_{i} C_{i, j} \hat{d}_{j}+\hat{d}_{i}^{\dagger} D_{i, j} \hat{d}_{j}^{\dagger}\right]$, where operators $\hat{d}_{i}$ are either bosonic or fermionic and vectors $\boldsymbol{i}=\left(i_{1}, \ldots, i_{D}\right) \in \mathcal{L}$ label individual sites of the cubic lattice. To ensure Hermiticity we demand the real coefficients to satisfy $A_{i, j}=B_{i, j}=A_{j, i}$ and $C_{i, j}=D_{i, j}=C_{j, i}$ for bosons, and $A_{i, j}=-B_{i, j}=A_{j, i}, \quad C_{i, j}=-D_{i, j}=$ $-C_{j, i}$ for fermions. Furthermore, we assume translational invariance and periodic boundary conditions (all coupling matrices depend only on the difference $\boldsymbol{i}-\boldsymbol{j}$ and are cyclic matrices). We will lead the discussion in terms of Hermitian operators $\hat{\boldsymbol{r}}=\left(\hat{x}_{1}, \ldots, \hat{x}_{|\mathcal{L}|}, \hat{p}_{1}, \ldots, \hat{p}_{|\mathcal{L}|}\right)^{t}$ (in a mild abuse of notation, the transposition refers to the tuple, not to operators) defined by $\hat{x}_{i}=\left(\hat{d}_{i}+\hat{d}_{i}^{\dagger}\right) / \sqrt{2}$ and $\hat{p}_{i}=$ $-i\left(\hat{d}_{i}-\hat{d}_{i}^{\dagger}\right) / \sqrt{2}$. In the bosonic case they are indeed position and momentum operators fulfilling the canonical commutation relations (CCR). In turn, for fermionic operators, they are so-called Majorana operators fulfilling the canonical anticommutation relations (CAR). We will assume isotropic couplings for fermions, $C=0$, and coupling only in position for bosons, $A-C=\mathbb{1}$, and we will not consider the straightforward but cumbersome generalization to anisotropic or momentum couplings. The Hamiltonian now reads $\left(V_{B}:=A+C, V_{F}:=A\right)$

$$
\hat{H}_{B}=\frac{1}{2} \hat{\boldsymbol{r}}^{t}\left[\begin{array}{cc}
V_{B} & 0 \\
0 & \mathbb{1}
\end{array}\right] \hat{\boldsymbol{r}}, \quad \hat{H}_{F}=\frac{i}{2} \hat{\boldsymbol{r}}^{t}\left[\begin{array}{cc}
0 & V_{F} \\
-V_{F}^{t} & 0
\end{array}\right] \hat{\boldsymbol{r}}
$$

for bosons and fermions, respectively. Whenever we may treat both species equally, we denote by $V$ the coupling in position $V=V_{B}$ for bosons and $V=V_{F}$ for fermions.

Starting from the Hamiltonians above, their spectrum and respective ground states are found by diagonalizing $\hat{H}_{B}$ through symplectic transformations, transformations respecting the CCR, and $\hat{H}_{F}$ by orthogonal transformations, transformations respecting the CAR. As matrices $V$ are cyclic, the bosonic spectrum is given by $\lambda_{k}$ and the fermionic spectrum by $\left|\lambda_{k}\right|$, where $\lambda_{k}=\sum_{l \in \mathcal{L}} V_{l} \cos (2 \pi k l / n)$, $k \in \mathcal{L}$.

The half-space geometry allows for a transformation of both Hamiltonians to a system of mutually uncoupled onedimensional chains while respecting the CCR and CAR, but notably, changing the local properties of the systems forming the individual chains. To this end consider the transformation $\hat{\boldsymbol{r}}=(\mathcal{O} \oplus \mathcal{O}) \hat{\boldsymbol{q}}$ to a new set of operators $\hat{\boldsymbol{q}}$, where the $|\mathcal{L}| \times|\mathcal{L}|$ matrix $\mathcal{O}$ is given by $\mathcal{O}_{i, j}=$ $\delta_{i_{1}, j_{1}} O_{i^{\prime}, j^{\prime}}$. Here and in the following we write vectors $\boldsymbol{i}=$ $\left(i_{1}, \ldots, i_{D}\right) \in \mathcal{L}$ as $\boldsymbol{i}=\left(i_{1} \boldsymbol{i}^{\prime}\right), \boldsymbol{i}^{\prime}=\left(i_{2}, \ldots, i_{D}\right) \in \mathcal{L}^{\prime}$. Now, the transformation $\mathcal{O}$ acts on the first coordinate as the identity and is thus local with respect to the bipartition $\mathcal{A} \mid \mathcal{B}$; i.e., it does not change entanglement properties. In order to respect the CAR and CCR the matrix $O$ needs to be orthogonal. Then, the Hamiltonians $\hat{H}_{B / F}$ read in coordinates $\hat{\boldsymbol{q}}$ just as in Eq. (1) with modified coupling matrices $V \mapsto \mathcal{O}^{t} V \mathcal{O}$. As cyclic matrices commute and may be diagonalized by the same Fourier transformation,

$$
V_{l}=\sum_{\boldsymbol{k}^{\prime} \in \mathcal{L}^{\prime}} \lambda_{\boldsymbol{k}^{\prime}}\left(l_{1}\right) e^{2 \pi i \boldsymbol{k}^{\prime} \cdot \boldsymbol{l}^{\prime} /\left|\mathcal{L}^{\prime}\right|} /\left|\mathcal{L}^{\prime}\right|
$$

$\lambda_{\boldsymbol{k}^{\prime}}\left(l_{1}\right)=\sum_{k_{1}=1}^{N} \lambda_{\left(k_{1} \boldsymbol{k}^{\prime}\right)} e^{2 \pi i k_{1} l_{1} / N} / N$, where the $\lambda_{\left(k_{1} \boldsymbol{k}^{\prime}\right)}=\lambda_{\boldsymbol{k}}$, $\boldsymbol{k} \in \mathcal{L}$, are the eigenvalues of $V$. Now, define the $\left|\mathcal{L}^{\prime}\right| \times$ $\left|\mathcal{L}^{\prime}\right|$ matrices $V\left(l_{1}\right)$ as those matrices obtained from $V$ by keeping the first coordinate fixed: $\left(V\left(l_{1}\right)\right)_{l^{\prime}}=V_{\left(l_{1} l^{\prime}\right)}$. Then the $\lambda_{\boldsymbol{k}^{\prime}}\left(l_{1}\right)$ are the eigenvalues of the $V\left(l_{1}\right)$, which are all cyclic and can thus all be diagonalized by the same orthogonal matrix. Choosing $O$ to be this matrix yields $\left(\mathcal{O}^{t} V \mathcal{O}\right)_{\left(i_{1} \boldsymbol{k}^{\prime}\right),\left(j_{1} \boldsymbol{p}^{\prime}\right)}=\delta_{\boldsymbol{k}^{\prime}, \boldsymbol{p}^{\prime}} \lambda_{\boldsymbol{k}^{\prime}}\left(i_{1}-j_{1}\right)$, a momentum space representation of the coupling in all but the first coordinate. In this representation the Hamiltonian is a sum of $\left|\mathcal{L}^{\prime}\right|$ mutually uncoupled one-dimensional chains labeled by $\boldsymbol{k}^{\prime}$. Each chain is described by a Hamiltonian of the form as in Eq. (1) with $N \times N$ cyclic coupling matrices $V\left(\boldsymbol{k}^{\prime}\right)$, $\left(V\left(\boldsymbol{k}^{\prime}\right)\right)_{l_{1}}=\lambda_{\boldsymbol{k}^{\prime}}\left(l_{1}\right)$. We will write $\left(V\left(\boldsymbol{\varphi}^{\prime}\right)\right)_{\phi_{1}}=\lambda_{\boldsymbol{\varphi}^{\prime}}\left(\varphi_{1}\right)$, $\phi_{1}=2 \pi l_{1} / N, \varphi_{d}^{\prime}=2 \pi k_{d}^{\prime} / N$, for the infinite system. The entanglement between $\mathcal{A}$ and $\mathcal{B}$ is now given by a sum of the entanglement between the sites $[1, \ldots, M]$ and $[M+1, \ldots, N]$ of the individual chains:

$$
\mathcal{E}=\lim _{N \rightarrow \infty} \sum_{\boldsymbol{k}^{\prime}} \frac{E_{S}\left(\boldsymbol{k}^{\prime}\right)}{N^{D-1}}=\int_{[0,2 \pi]^{\times D-1}} E_{S}\left(\boldsymbol{\varphi}^{\prime}\right) \frac{d \boldsymbol{\varphi}^{\prime}}{(2 \pi)^{D-1}} .
$$

This will be the starting point for the following discussion.

Fermions. - We start with investigating case (ii) above: The asymptotic behavior in $M$ after taking the limit $N \rightarrow$ $\infty$ [20], frequently referred to as the double scaling limit [17]. For each chain $\varphi^{\prime}$ we now need to compute the entanglement between the first $M$ sites and the rest of the chain (in real space, not momentum space). The asymptotic behavior in $M$ of this entanglement can be obtained from the so-called symbol $g_{\varphi^{\prime}}\left(\varphi_{1}\right)=\operatorname{sgn}\left(\lambda_{\varphi^{\prime}}\left(\varphi_{1}\right)\right)$ of the chain $[17,18]$ : Each $E_{S}\left(\boldsymbol{\varphi}^{\prime}\right)$ is determined from the continuity properties of $g_{\varphi^{\prime}}$ as a function of $\varphi_{1}$. For fixed $\varphi^{\prime}$ it corresponds to a one-dimensional isotropic fermionic model, for which the asymptotic form of the entanglement has been obtained in Refs. [17],

$$
E_{S}\left(\boldsymbol{\varphi}^{\prime}\right)=\frac{s\left(\boldsymbol{\varphi}^{\prime}\right)}{6} \log _{2} M+c\left(\boldsymbol{\varphi}^{\prime}\right)+o(\log M),
$$

where the function $c\left(\varphi^{\prime}\right)$ does not depend on $M$ and the 
integer $s\left(\boldsymbol{\varphi}^{\prime}\right)$ is the number of discontinuities of $g_{\boldsymbol{\varphi}^{\prime}}$ as function of $\varphi_{1}$ in the interval $[0,2 \pi)$. All chains with $s\left(\boldsymbol{\varphi}^{\prime}\right)>0$ are critical as finding discontinuities in the symbol is equivalent to having a vanishing energy gap above the ground state. This means that, depending on the Fermi surface (the set of solutions to $\lambda_{\varphi}=0$; see Fig. 1), one finds a continuum of chains $\boldsymbol{\varphi}^{\prime}$ that are critical. This situation is in contrast to the situation encountered when considering bosonic systems, as we will see below. From (2), we find the asymptotic behavior in $M$ as

$$
\mathcal{E}=\frac{\log _{2} M}{6} \sum_{\sigma=1}^{\infty} \frac{\sigma v\left(\Phi_{\sigma}\right)}{(2 \pi)^{D-1}}+\int \frac{d \boldsymbol{\varphi}^{\prime} c\left(\boldsymbol{\varphi}^{\prime}\right)}{(2 \pi)^{D-1}}+o(\log M),
$$

where we defined $v\left(\Phi_{\sigma}\right)=\int_{\Phi_{\sigma}} d \varphi^{\prime}$ as the volume of the set $\Phi_{\sigma}=\left\{\boldsymbol{\varphi}^{\prime}: s\left(\boldsymbol{\varphi}^{\prime}\right)=\sigma\right\}$, so the set associated with exactly $\sigma$ discontinuities. Hence, we do encounter a $\log a$ rithmic divergence in $M$ of the entanglement entropy and the prefactor depends on the topology of the Fermi surface: $g_{\varphi^{\prime}}$ exhibits discontinuities at points where $\lambda_{\varphi}=0$, i.e., on the Fermi surface. If the Fermi surface is of measure zero (i.e., the set of solutions to $\lambda_{\varphi}=0$ is countable, as, for example, in the critical bosonic case; see Fig. 1), we have $v\left(\Phi_{\sigma}\right)=0$ and the system obeys the area law, $\mathcal{E}=$ const. Consider as an example the case of a nearest-neighbor Hamiltonian with coupling $V_{i, j}=\delta_{i, j}+a \delta_{\text {dist }(i, j), 1}$, in which case the symbol corresponds for fixed $\boldsymbol{\varphi}^{\prime}$ to that of the isotropic $X Y$ model with transverse magnetic field $h\left(\boldsymbol{\varphi}^{\prime}\right)=1+2 a \sum_{d} \cos \left(\varphi_{d}^{\prime}\right)$. For this model, the nonleading-order term was obtained employing FisherHartwig-type methods [17]. It reads $c\left(\varphi^{\prime}\right)=\log _{2}(1-$ $\left.h^{2}\left(\boldsymbol{\varphi}^{\prime}\right) / 4\right) / 6+c_{0}$ and vanishes if the chain $\boldsymbol{\varphi}^{\prime}$ is noncritical. The constant $c_{0}$ is independent of the system parameters. The number of discontinuities is $s\left(\varphi^{\prime}\right)=2$ for
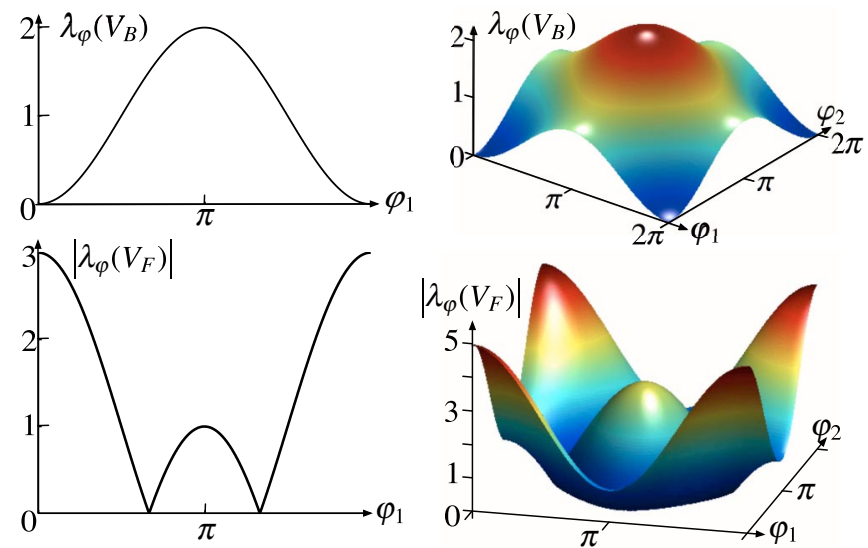

FIG. 1 (color online). Spectra of critical nearest-neighbor Hamiltonians (top: bosonic; bottom: fermionic) in one and two dimensions. The spectrum of the individual decoupled chains is given by the spectrum of the full Hamiltonian along the first coordinate $\varphi_{1}$. The topology of the set of solutions to $\lambda_{\varphi}=0-$ the Fermi surface in the fermionic case-provides an intuition as to why the scaling behavior of entanglement is different for fermions then for bosons in $D>1$; see text. $\boldsymbol{\varphi}^{\prime} \in \Phi_{2}=\left\{\boldsymbol{\varphi}^{\prime} \in[0,2 \pi)^{D-1}:\left|\frac{1}{2 a}+\sum_{d} \cos \left(\varphi_{d}^{\prime}\right)\right|<1\right\}$ and zero otherwise; i.e., the sum over $\sigma$ in (3) consists only of the $\sigma=2$ term as all others are zero. Thus

$$
\mathcal{E}=\frac{v\left(\Phi_{2}\right)}{3(2 \pi)^{D-1}} \log _{2} M+\int_{\Phi_{2}} \frac{c(\boldsymbol{\varphi}) d \boldsymbol{\varphi}^{\prime}}{(2 \pi)^{D-1}}+o\left(\log _{2} M\right),
$$

where for $D=2$ and the critical case $|a|>1 / 4$, we find $v\left(\Phi_{2}\right)=2 \arccos [1 /(2|a|)-1]$; i.e., the prefactor depends on the coupling parameter $a$. For noncritical models in the isotropic setting at hand the set $\Phi_{2}$ is empty and there is no entanglement. There is no universal non-leading-order term as proposed in Ref. [8] related to the conformal charge, due to this specific geometric setting.

At this point, it is interesting to discuss the behavior of the entanglement entropy under Lifshitz phase transitions. They are topological quantum phase transitions of fermionic systems due to a change of the topology of the Fermi surface, occurring, for example, in $d$-wave superconductors $[1,13]$. The previous considerations allow us to argue that a Lifshitz transition accompanied by a change of topology of the Fermi surface is reflected by a nonanalyticity in the prefactor $v\left(\Phi_{\sigma}\right)$ of the entanglement scaling law: Any change of the topology will lead to a nondifferentiable alteration of the prefactor of the leading order term.

The second setting is the one of $M=N / 2$ [case (i) above], where - in contrast to the double scaling limit $M$ depends on $N$, leaving one limit to consider. From the discussion above, we would expect $\mathcal{E}=\infty$. Indeed, making use of a quadratic lower bound to the entanglement entropy $[7,16]$, it can be shown that fermions violate the area law in this setting: For fermionic models with nearestneighbor interactions with half-filling in $D=2$, so $V_{i, j}=$ $a \delta_{d(i, j), 1}$, we find after some straightforward algebra [21] $\lim _{N \rightarrow \infty} \sum_{k=1}^{N} E_{S}(k) / N \geq \lim _{N \rightarrow \infty} \sum_{l=1}^{N / 2-1} 4 /\left(\pi^{2} l\right)=\infty$.

Bosons. - We concentrate on the geometrical setting $M=N / 2$ [case (i) above] and the most significant model: The $D$-dimensional free Klein Gordon field,

$$
\hat{H}=\frac{1}{2} \int_{[0, L]^{\times D}} d \boldsymbol{r}\left[\pi(\boldsymbol{r})^{2}+\mu^{2} \phi(\boldsymbol{r})^{2}+v^{2}(\boldsymbol{\nabla} \phi(\boldsymbol{r}))^{2}\right],
$$

which may be obtained from a Hamiltonian as in Eq. (1) with a nearest-neighbor coupling $V_{i, j}=$ $\left(\mu^{2}+2 D \Omega^{2}\right) \delta_{i, j}-\Omega^{2} \delta_{d(i, j), 1}$ : Denoting the lattice spacing by $\alpha=L / N$ and taking the limit $N \rightarrow \infty$ while keeping $v^{2}=\Omega^{2} \alpha^{2}$ constant, one obtains (4). Rescaling $V \mapsto$ $V /\left(\mu^{2}+2 D \Omega^{2}\right)$, we find $V_{i, j}=\delta_{i, j}-c_{N} \delta_{d(i, j), 1}$ and $c_{N}=\left[\mu^{2} L^{2} /\left(v^{2} N^{2}\right)+2 D\right]^{-1} \rightarrow_{N \rightarrow \infty} 1 / 2 D$. Now, demanding the system to be critical uniquely determines $c_{N} \rightarrow 1 /(2 D)$ as the energy gap between the ground and first excited state is given by the square root of the smallest eigenvalue of the coupling matrix $V$.

For each individual chain $\boldsymbol{k}^{\prime}$, the $V\left(\boldsymbol{k}^{\prime}\right)$ are transformed nearest-neighbor coupling matrices: $\left(V\left(\boldsymbol{k}^{\prime}\right)\right)_{i, j}=$ $\left[1-2 c_{N} \sum_{d} \cos \left(2 \pi k_{d}^{\prime} / N\right)\right] \delta_{i, j}-c_{N} \delta_{d(i, j), 1}$. In the infinite 
system limit, the energy gap $\Delta E\left(\varphi^{\prime}\right)$ between the ground and first excited state of each chain is given by $\Delta E\left(\varphi^{\prime}\right)^{2} D=D-\sum_{d} \cos \left(\varphi_{d}^{\prime}\right)-1$. Hence, in contrast to the critical fermionic case, the set of solutions to $\lambda_{\varphi}=0$ is of measure zero as only a single chain becomes critical, when we identify 0 with $2 \pi$ in the spectrum; see Fig. 1 . We now make use of a powerful result of Ref. [4]: The exact form of the logarithmic negativity $E_{n}$ (an upper bound to the entropy of entanglement and an entanglement monotone $[22,23])$ with respect to the split $[1, \ldots, N / 2] \mid[N / 2+$ $1, \ldots, N]$ for a harmonic chain with nearest-neighbor coupling. It is not only an asymptotic statement in $N$, but a closed-form expression and we find that $\lim _{N \rightarrow \infty} \sum \boldsymbol{k}^{\prime} E_{n}\left(\boldsymbol{k}^{\prime}\right) / N^{D-1}$ converges to

$\frac{1}{2(2 \pi)^{D-1}} \int_{[0,2 \pi]^{D-1}} d \boldsymbol{\varphi}^{\prime} \log _{2}\left(\frac{D-\sum_{d=1}^{D-1} \cos \left(\varphi_{d}^{\prime}\right)+1}{D-\sum_{d=1}^{D-1} \cos \left(\varphi_{d}^{\prime}\right)-1}\right) \geq \mathcal{E}$,

independent of the mass $\mu$. For $D=2$ it evaluates to $\log _{2}(3+2 \sqrt{2}) / 2$ and similarly for $D>2$. Hence, the entanglement entropy for this critical model is bounded by an expression linear in the boundary area, we do not encounter an infrared divergence here, and the prefactor can be exactly determined for the logarithmic negativity.

Summary. - In this work, we have clarified the issue of scaling of the entanglement entropy in bosonic and fermionic lattice systems. Our analytical argument indeed confirms and resolves previous numerical findings and conjectures on the scaling of entanglement in ground states of many-body systems. The difference between the behavior of bosons and fermions may be taken as unexpected. The violation of the area law for fermions is in fact intertwined with the specific role of the Fermi surface. We found that quantum phase transitions involving an alteration of the topology of the Fermi surface result in a nonanalytical behavior of the prefactor.

For typical critical models we found that those individual boundary crossing chains obtained from our decoupling procedure that are critical form a continuum for fermions and are finite in number for bosons. Integrating over all chains then "lifts" singularities in $D>1$ for bosons. For critical bosonic models exhibiting only a finite number of ground states, one might expect that an area law holds in dimensions $D>1$ even for models that go beyond quadratic Hamiltonians considered here and are thus truly interacting. Confirming or refuting this conjecture is an interesting challenge.

This work has benefited from discussions with M. M. Wolf, T. J. Osborne, M. Ericsson, and M. B. Hastings. It has been supported by the DFG (No. SPP 1116), the EU (QAP), the Royal Society, the QIP-IRC, Microsoft Research, and EURYI.

[1] M. Vojta, Rep. Prog. Phys. 66, 2069 (2003).

[2] M. Srednicki, Phys. Rev. Lett. 71, 666 (1993); L. Bombelli et al., Phys. Rev. D 34, 373 (1986); C. Callan and
F. Wilczek, Phys. Lett. B 333, 55 (1994); A. Riera and J. I. Latorre, Phys. Rev. A 74, 052326 (2006).

[3] M.-C. Chung and I. Peschel, Phys. Rev. B 62, 4191 (2000).

[4] K. Audenaert et al., Phys. Rev. A 66, 042327 (2002).

[5] M. B. Plenio et al., Phys. Rev. Lett. 94, 060503 (2005).

[6] M. Cramer et al., Phys. Rev. A 73, 012309 (2006); M. Cramer and J. Eisert, New J. Phys. 8, 71 (2006).

[7] M. M. Wolf, Phys. Rev. Lett. 96, 010404 (2006); D. Gioev and I. Klich, ibid. 96, 100503 (2006).

[8] A. Kitaev and J. Preskill, Phys. Rev. Lett. 96, 110404 (2006); E. Fradkin and J.E. Moore, ibid. 97, 050404 (2006).

[9] J. Eisert and T. J. Osborne, Phys. Rev. Lett. 97, 150404 (2006); S. Bravyi, M. B. Hastings, and F. Verstraete, ibid. 97, 050401 (2006); G. De Chiara et al., J. Stat. Mech. (2006) P03001.

[10] F. Verstraete and J.I. Cirac, Phys. Rev. B 73, 094423 (2006); T. J. Osborne, Phys. Rev. Lett. 97, 157202 (2006); J. Eisert, ibid. 97, 260501 (2006).

[11] F. Verstraete et al., Phys. Rev. Lett. 96, 220601 (2006).

[12] T. Barthel, M.-C. Chung, and U. Schollwöck, Phys. Rev. A 74, 022329 (2006).

[13] W. Li et al., Phys. Rev. B 74, 073103 (2006).

[14] R. G. Unanyan and M. Fleischhauer, Phys. Rev. Lett. 95, 260604 (2005).

[15] G. Vidal et al., Phys. Rev. Lett. 90, 227902 (2003); P. Calabrese and J. Cardy, J. Stat. Mech. (2004) P06002; I. Peschel, J. Stat. Mech. (2004) P12005; A. Botero and B. Reznik, Phys. Rev. A 70, 052329 (2004); T. Barthel, S. Dusuel, and J. Vidal, Phys. Rev. Lett. 97, 220402 (2006); R. G. Unanyan, M. Fleischhauer, and D. Bruss, Phys. Rev. A 75, 040302 (2007); S. Das and S. Shankaranarayanan, Phys. Rev. D 73, 121701 (2006); H. Casini and M. Huerta, Nucl. Phys. B764, 183 (2007).

[16] M. Fannes, B. Haegeman, and M. Mosonyi, J. Math. Phys. (N.Y.) 44, 6005 (2003).

[17] J. P. Keating and F. Mezzadri, Phys. Rev. Lett. 94, 050501 (2005); A. R. Its, B.-Q. Jin, and V. E. Korepin, J. Phys. A 38, 2975 (2005).

[18] J. Eisert and M. Cramer, Phys. Rev. A 72, 042112 (2005).

[19] M. Hein, J. Eisert, and H.J. Briegel, Phys. Rev. A 69, 062311 (2004); A. Hamma, R. Ionicioiu, and P. Zanardi, ibid. 71, 022315 (2005); W. Dür et al., Phys. Rev. Lett. 94, 097203 (2005).

[20] Considering the entanglement of a single site with the rest, we find $E_{S}=\left(\varrho_{F}-1\right) \log _{2}\left(1-\varrho_{F}\right)-\varrho_{F} \log _{2}\left(\varrho_{F}\right)$, which is equal to zero for noncritical systems $\left(\lambda_{k}<0\right.$ or $\left.\lambda_{k}>0\right)$, maximal for half-filling, and independent of $N$, thereby allowing for a meaningful study of the scaling of entanglement in $M$ after letting $N \rightarrow \infty$. Here, the filling factor is given by $\varrho_{F}=\frac{1}{2}-\sum_{k} \operatorname{sgn}\left(\lambda_{k}\right) /\left(2 N^{D}\right)$. For critical bosons in $D=1$ this entanglement diverges in $N$; i.e., the scaling in the size of $\mathcal{A}$ after letting $N \rightarrow \infty$ is not well defined - a manifestation of the infrared divergence in $D=1[2]$.

[21] The quadratic lower bound explicitly evaluates to $E_{S}(k) \geq$ $\left(4 / N^{2}\right) \sum_{i=1}^{N / 2} \sum_{j=N / 2+1}^{N} \csc ^{2}[(i-j) \pi / N] \sin ^{2}[(i-1)(1-$ $2 k) \pi / N]$.

[22] G. Vidal and R.F. Werner, Phys. Rev. A 65, 032314 (2002).

[23] J. Eisert, Ph.D. thesis, University of Potsdam, 2001; M. B. Plenio, Phys. Rev. Lett. 95, 090503 (2005). 